\title{
Treatment of Chronic Hepatitis C in Treatment-Naïve Patients
}

\author{
Marcelo Simão Ferreira \\ Federal University of Uberlândia School of Medicine; Uberlândia, MG, Brazil
}

\begin{abstract}
After the initial acute phase of infection, $50-85 \%$ of patients infected with the hepatitis $\mathrm{C}$ virus (HCV) develop the chronic form of the disease, which, in 20-30\% of cases, will evolve to cirrhosis, liver failure or hepatocellular carcinoma, albeit after several decades. Once this infection has been established, it rarely resolves spontaneously. It is known that, during the chronic phase, the more severe forms of this viral infection can be induced by various cofactors: chronic alcoholism; coinfection with HIV or the hepatitis B virus; liver biopsy-proven steatosis (or steatohepatitis); and advanced age. The main objective for treating this disease is, therefore, to prevent the occurrence of late complications, by means of the eradication of HCV, which can be achieved in just over half of the cases treated with the currently available drugs.
\end{abstract}

\section{Treatment for Chronic Hepatitis C: Drugs and Treatment Response Patterns}

The currently recommended treatment for the chronic forms of hepatitis $\mathrm{C}$ is the combination of interferon alpha (IFN- $\alpha$ ) and ribavirin. The former is a cytosine that is a component of the innate response of the human host. Various genes involved in the immune response are induced/stimulated by IFN- $\alpha$, resulting in the activation of natural killer cells, maturation of dendritic cells, and proliferation of memory cells, as well as in the prevention of apoptosis of $\mathrm{T}$ cells. The hepatocellular injury seen in chronic hepatitis $\mathrm{C}$ is not due to the cytopathic effect of HCV. It is immunomediated by natural killer cells and CD8 T lymphocytes, which are activated by the action of IFN- $\alpha$.

Ribavirin is an oral nucleoside analog with antiviral effects against various pathogens (respiratory syncytial virus, arenavirus, etc.) Although its mechanism of action in HCV remains unclear, it seems that ribavirin causes the virus to mutate rapidly to forms that are more easily killed, as well as depleting intracellular adenosine triphosphate, which is essential for the synthesis of viral RNA. Immunomodulatory effects have been also attributed to this drug.

The use of IFN- $\alpha$ was approved for hepatitis C treatment in 1991. At that time, the response to this immunotherapy was very low $(<20 \%)$. However, when the combination of ribavirin and IFN- $\alpha$ began to be used, the proportion of patients in whom a sustained virological response (SVR) was achieved grew to $40-45 \%$. Even at that time, treatment responses were confirmed by detecting HCV RNA through molecular tests such as polymerase chain reaction (PCR). In this context, the infection is considered eradicated when there is an SVR, which is defined as the absence of serum HCV RNA in a sensitive test (qualitative PCR) at the end of treatment and at six months

The Brazilian Journal of Infectious Diseases $\quad$ 2007;11 (5) Suppl. 1:45-49. (C) 2007 by The Brazilian Journal of Infectious Diseases and Contexto Publishing. All rights reserved. after the end of treatment. Patients who achieve an SVR almost always show a dramatic decrease in HCV RNA levels, defined as a $\geq 2 \log _{10}$ drop or the absence of HCV RNA by 12 weeks after the initiation of treatment. This response is designated the early virological response (EVR) and has been widely used for treatment follow-up of patients infected with HCV genotype 1 . The maintenance of the undetectable viral load status at the end of the treatment is designated the end-oftreatment response (ETR). A patient is considered recidivist when HCV RNA becomes undetectable during treatment but becomes positive again after the end of the treatment, whereas a patient is considered a nonresponder when HCV RNA levels remain stable or decrease $<2 \log _{10}$ during treatment with the combined regimen.

The most recent advance in the treatment of hepatitis $C$ has been the development of long-acting, pegylated formulations of IFN- $\alpha$ (PEG-IFN- $\alpha$ ), produced by the covalent addition of a polyethylene glycol molecule to the IFN- $\alpha$ molecule. This combination decreased absorption, reduced the clearance of the drug and increased its half-life. With this increased half-life, PEG-IFN- $\alpha$ can be administered in weekly doses. Two pegylated formulations have now been approved for the treatment of hepatitis C: PEG-IFN- $\alpha$ 2a (PegasysRoche), with a molecular weight of $40 \mathrm{kDa}$, and PEG-IFN- $\alpha 2 \mathrm{~b}$ (PEG-INTRON; Schering-Plough), with a molecular weight of $12 \mathrm{kDa}$.

Table 1 shows the names and doses of the drugs that are currently used for the treatment of chronic hepatitis C.

\section{Viral Kinetics After the Beginning of Treatment}

The most important objective of chronic hepatitis $\mathrm{C}$ treatment is HCV eradication. The introduction of treatment leads to a biphasic drop in the viral population. The speed at which the quantity of HCV drops differs among the treated patients, and the drops can therefore be classified as rapid or slow. Patients in whom there are rapid drops at the beginning of treatment more often achieve an SVR. During this rapid response phase, which generally occurs within the first $48 \mathrm{~h}$ of HCV treatment, the viral load decreases rapidly, which reflects the IFN- $\alpha$ inhibition of replication and the degradation of the drug in the serum. The HCV RNA titers begin to decline 8 to $12 \mathrm{~h}$ after the administration of the first IFN- $\alpha$ dose, and the drop ranges from 0.5 to $1.5 \log _{10}$ within the first $48 \mathrm{~h}$. Thereafter, the rate of viral load reduction slows, reflecting the clearance of the virus in the infected cells. The complete elimination of viral particles requires combined treatment for several months. During this second phase, ribavirin seems to play a crucial role in $\mathrm{HCV}$ depuration. Negative HCV viral load during treatment is generally followed by alanine aminotransferase normalization and improvement in the necroinflammatory activity in liver biopsy. 
Table1. Drugs used for the treatment of chronic hepatitis C

\begin{tabular}{|c|c|}
\hline Drugs & Recommended doses \\
\hline \multicolumn{2}{|l|}{ Conventional interferon } \\
\hline Alpha 2a & 3 MU sc $3 \times$ week \\
\hline Alpha 2b & 3 MU sc $3 \times$ week \\
\hline Consensus & $9 \mu \mathrm{g}$ sc $3 \times$ week \\
\hline \multicolumn{2}{|l|}{ Pegylated interferon } \\
\hline Peginterferon $\alpha$ 2a (40 kDa) & $180 \mu \mathrm{g}$ SC / week \\
\hline Peginterferon $\alpha$ 2b (12 kDa) & $1.5 \mu \mathrm{g} / \mathrm{kg} \mathrm{SC} /$ week \\
\hline \multicolumn{2}{|l|}{ Nucleoside analogs } \\
\hline Ribavirin & $\begin{array}{l}\text { From } 1,000 \mathrm{mg}(\leq 75 \mathrm{~kg}) \text { to } \\
1250 \mathrm{mg}(>75 \mathrm{~kg}) \text {, oral, daily }\end{array}$ \\
\hline
\end{tabular}

MU: million units; sc: subcutaneous.

\section{Treatment Protocols}

For adults diagnosed with chronic hepatitis $\mathrm{C}$ and presenting detectable serum levels of HCV RNA, together with persistent elevation of aminotransferases, histological evidence of progressive hepatic disease, no severe comorbidities, and no contraindications, treatment is recommended. All patients should be initially submitted to viral load quantification (quantitative PCR), HCV genotype identification (genotypes 1 to 6) and liver biopsy for the evaluation of necroinflammatory activity (intensity) and fibrosis (staging). The two most common methods for histological evaluation are the METAVIR and Ishak scoring systems, in which fibrosis is scored as absent (F0), only portal (F1), portal with septum formation (F2), hepatic with portalcentral and portal-portal bridging (F3), or cirrhosis (F4). Treatment is recommended for patients who present a score of at least F2.

It must be borne in mind that liver biopsy is an invasive, costly, and potentially fatal procedure. For patients presenting HCV genotype 1, this procedure is useful for therapeutic decisions when there is no evidence of advanced fibrosis detected by other methods (ultrasound, etc.) However, for those presenting genotype 2 or 3 and high treatment response rates, liver biopsy might be unnecessary and might not influence the therapeutic decision, especially for those individuals who present persistently high levels of aminotransferases.

The combination of PEG-IFN- $\alpha$ and ribavirin is the treatment currently recommended for patients with chronic hepatitis C; its efficacy in achieving an SVR is greater than that of the conventional treatment with IFN- $\alpha$ in isolation or with the combination of IFN- $\alpha$ and ribavirin (56\% vs. $16 \%$ vs. 42\%). As demonstrated in Table 1, subcutaneous PEG-IFN-a is administered weekly, and oral ribavirin is administered in two daily doses. The recommended dose of PEG-IFN- $\alpha$ 2a is $180 \mu \mathrm{g}$ per week and that of PEG-IFN- $\alpha$ 2b is $1.5 \mu \mathrm{g} / \mathrm{kg}$ per week. Treatment duration and ribavirin dosing vary according to HCV genotype. Patients presenting HCV genotype 1 must be treated with either 1,000-mg or 1,250-mg doses of ribavirin (body weight $\leq 75 \mathrm{~kg}$ or $>75 \mathrm{~kg}$, respectively) for 48 weeks. Patients infected with HCV genotype 2 or 3 must receive 800 mg of ribavirin daily for 24 weeks. In Brazil, ribavirin capsules contain $250 \mathrm{mg}$, and so it is not possible to administer the recommended 800-mg doses. Therefore, we believe it is prudent to prescribe 1000-mg doses for all patients infected with these genotypes. There is little information on the treatment against hepatitis $C$ genotypes 4,5 , and 6 (which are rare in Brazil). It has been recommended that the 48-week treatment regimen be used in these cases.

In the treatment for infection with HCV genotype 1, the possibility of achieving an SVR is based on the EVR, as previously defined. Negative results or a significant $\left(\geq 2 \log _{10}\right)$ drop in HCV viral load by week 12 of treatment is indicative of an SVR, which appears in $65 \%$ of the patients treated with PEG-IFN- $\alpha$ 2a and in 72\% of those treated with PEG-IFN- $\alpha 2 b$. However, among those who have not achieved an EVR by week 12 using either formulation, only 3\% achieve an SVR. Therefore, the EVR is a strong negative predictor of the SVR. All patients who continue to present positivity (based on viral loads) at week 12 should be re-assessed at week 24 , at which point a qualitative PCR should be performed. If results are negative, treatment should be maintained up to week 48 . If is the results are still positive, treatment should be discontinued. For HCV genotypes 2 and 3, it is not generally recommended that molecular tests be performed during the 24-week treatment regimen. At the end of the combined treatment, regardless of the genotype, a qualitative PCR should be performed in order to evaluate the ETR.

Chart 1 shows the algorithm for the follow-up of patients with chronic hepatitis $C$ during the treatment with PEG-IFN- $\alpha$ and ribavirin.

With the current treatment protocols, the rate at which an SVR is achieved ranges from 54\% to $56 \%$ after the use of the combination of PEG-IFN- $\alpha$ (either formulation) and ribavirin, which is considerably higher than that seen with older conventional treatments (Chat 1). Among patients infected with HCV genotype 2 or 3, the rate at which an SVR is achieved ranges from $75 \%$ to $80 \%$, compared with $40 \%$ to $61 \%$ among those infected with HCV genotype 1. Among patients with HCV genotype 1, the rate at which an SVR is achieved is lower in blacks (28\%) than in Caucasians (52\%). Other factors that influence treatment response, leading to lower cure rates, are high viral load levels (> 600,000 IU/mL), male gender, high body mass index, biopsy showing advanced fibrosis and high iron levels in the hepatic parenchyma. In patients infected with HCV genotype 2 or 3 and treated with a conventional regimen involving the combination of IFN- $\alpha$ (3 million units 3 times a week) and ribavirin, the rate at which an SVR is achieved can be similar to that of those treated with pegylated formulations and ribavirin. Therefore, this regimen is still recommended in Brazil (directive 863/2002). For such patients, the use of this treatment regimen for six months is less costly and better tolerated.

Patients with liver cirrhosis have lower chances to eliminate HCV by means of the current treatment than do noncirrhotic patients. This might be due to the fact that the former present more advanced age, greater alterations in hepatic microcirculation (which prevent the adequate interaction between IFN and infected cells), and lower treatment 
Chart 1. Chronic hepatitis treatment strategies

Treatment for infection with HCV genotype 1: evolution dynamics

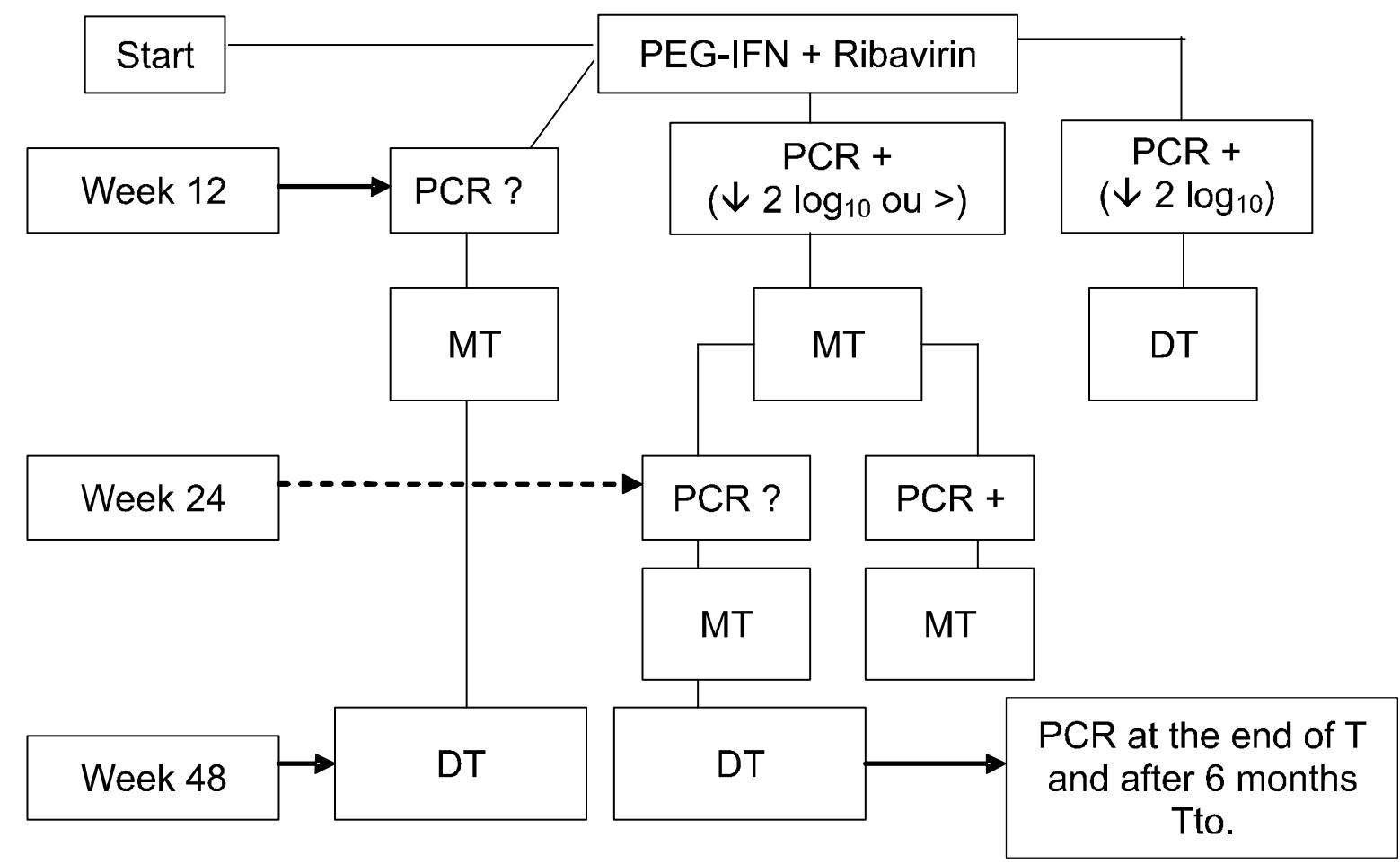

PEG-IFN=pegylated interferon

$\mathrm{PCR}=$ polymerase chain reaction

$\mathrm{MT}=$ maintain treatment

DT=discontinue treatment

$\mathrm{T}=$ treatment

Table 2. Drugs for the treatment of chronic hepatitis C

\begin{tabular}{|c|c|c|c|c|c|}
\hline Genotype & $\begin{array}{l}\text { PEG-IFN- } \alpha \\
\text { (dose) }\end{array}$ & Ribavirin & $\begin{array}{l}\text { Duration } \\
\text { (dose) }\end{array}$ & $\begin{array}{l}\text { Treatment evolution } \\
\text { (weeks) }\end{array}$ & $\begin{array}{l}\text { SVR } \\
(\%)\end{array}$ \\
\hline $\begin{array}{c}1 \\
(4,5,6)\end{array}$ & $\begin{array}{l}\alpha \text { 2a: } 180 \mu \mathrm{g} / \text { week } \\
\alpha \text { 2b: } 1.5 \mu \mathrm{g} / \mathrm{kg} / \text { week }\end{array}$ & $\begin{array}{l}1.0 \mathrm{~g}(<75 \mathrm{~kg}) \\
1.25 \mathrm{~g}(>75 \mathrm{~kg})\end{array}$ & 48 & $\begin{array}{l}\text { a) No response: }{ }^{-} \text {HCV RNA at week } 12 \\
<2 \log _{10} \text { IU/mL or HCV RNA } \oplus \text { by week } 24 \\
\text { b) Rapid response: treat for } 24 \text { weeks if } \\
\text { HCV RNA } \Theta \text { by week } 4 \text { and low initial } \\
\text { viral load }(<600,000 \mathrm{IU} / \mathrm{mL})\end{array}$ & $40-61$ \\
\hline 2,3 & Same as above & $1.0 \mathrm{~g}$ & 24 & $\begin{array}{l}\text { a) No response: uncommon } \\
\text { b) Rapid response: treat for 12-16 weeks } \\
\text { if HCV RNA } \Theta \text { by week } 4\end{array}$ & $75-80$ \\
\hline
\end{tabular}

PEG-IFN=pegylated interferon; SVR=sustained virological response; HCV=hepatitis C virus. 
compliance rates. These patients should be treated carefully due to the risk of decompensation of the disease during treatment and the worsening of pre-existing hematological parameters (leukopenia and platelet reduction). Patients with compensated cirrhosis should be treated if they present the minimum criteria to receive these medications:

a) Total bilirubin $<1.5 \mathrm{~g} / \mathrm{dL}$

b) Albumin $>3.4 \mathrm{~g} / \mathrm{dL}$

c) Platelets $>75,000 / \mathrm{mm}^{3}$

d) Hemoglobin $>13 \mathrm{~g} / \mathrm{dL}$; neutrophils $>1500 / \mathrm{mm}^{3}$

e) Creatinine $<1.5 \mathrm{mg} / \mathrm{dL}$

f) Absence of ascites, together with hepatic encephalopathy. In patients with advanced fibrosis and treated with the PEG-IFN- $\alpha$ /ribavirin combination, the rate at which an SVR is achieved ranges from $37 \%$ to $50 \%$, being higher (70\%-75\%) in those presenting HCV genotype 2 or 3, as well as in those with low viral loads. Side effects, especially thrombocytopenia and neutropenia, are more common in these patients. At this stage of the disease, cirrhotic patients who achieved an SVR are not totally protected against the risk of developing hepatocellular carcinoma. Two recent studies have confirmed a limited reduction in the risk of hepatocellular carcinoma in patients who successfully responded to the combined regimen; however, some degree of risk remains due to the carcinogenic effect of hepatic fibrosis. In addition, the complications of advanced liver disease, in these cases, occur less frequently, mortality is lower, and there will obviously be no re-infection of the organ after the liver transplant. In order to prevent re-infection in transplanted patients, those who are on a liver transplant waiting list and present decompensated cirrhosis have been treated with IFN- $\alpha$ or PEG-IFN- $\alpha$ and ribavirin for periods ranging from three to fourteen months. In these individuals, the rate at which an SVR is achieved has been low (from 20\% to 25\%), and morbidity/mortality due to the treatment have been considerably high. Bacterial infections, severe cytopenias, and even mortality have been reported during treatment. However, in those patients who responded to treatment, with the elimination of HCV, there was improvement in liver function, fewer episodes of decompensation and lower mortality. Benefits seem to be higher, as expected, for patients infected with HCV genotype 2 or 3, who traditionally respond better to the treatment regimen.

Not every patient whose HCV RNA levels become undetectable during treatment achieves an SVR. In 10\% of the treated patients, this molecular marker reappears in the serum during treatment, whereas in $20 \%$ of the treated patients, it reappears after the end of treatment (recurrence); in this context, HCV RNA becomes detectable a few weeks after the interruption of treatment, and aminotransferase levels again increase. Recurrence is more common with short treatment or when there is a delay in achieving negative HCV RNA results. In those patients who responded to treatment and achieved an SVR, long-term follow-up evaluations have shown that HCV RNA results remain negative in more than 95\% of these patients, confirming the cure and the improvement in histological parameters.

\section{Modifications During the Course of Treatment of Chronic Hepatitis C}

In patients infected with HCV genotype 1, a 72-week combined regimen (PEG-IFN- $\alpha+$ ribavirin) may be beneficial for slow responders, who fail to present negative HCV RNA results by week 4 or week 12 of treatment (EVR). In a randomized study, 326 patients still presented positive results for the virus at week 4 of treatment. These patients received the medications for either 48 or 72 weeks. The rate at which an SVR was achieved was significantly higher in the group receiving the longer-duration regimen ( $45 \%$ vs. $32 \%$; $\mathrm{p}=0.01$ ). In another randomized study, patients who did not achieve an EVR were submitted to either 48 or 72 weeks of treatment. The results showed that $29 \%$ (31/106) of the patients in the 72 week group achieved an SVR, compared with only 17\% (17/ $100)$ of those in the 48-week group ( $p=0.04)$. Therefore, selected patients who present slow initial response to antiviral medications can be treated for longer periods.

However, the treatment duration can be shortened if patients develop a rapid virological response (RVR), defined as presenting a response by week 4 of treatment. Various studies have demonstrated that negative results in the qualitative PCR by week 4 of treatment have a positive predictive value for SVR. Studies involving patients infected with HCV genotype 2 or 3 who achieve an RVR with the use of PEG-IFN- $\alpha 2 \mathrm{~b}$ and ribavirin have demonstrated that treatment can be discontinued at 12 to 16 weeks after the beginning of treatment, since these patients presented SVR rates that were similar to those seen in the control group, which was submitted to the 24-week regimen. Recurrences were especially more common among patients infected with HCV genotype 2 and in those with high viral loads before treatment. In a recent randomized study, 150 patients infected with HCV genotype 2 were treated with PEG-IFN- $\alpha$ and ribavirin for either 16 or 24 weeks. The comparison showed that both groups presented high SVR rates (94\% and 95\%, respectively), indicating that patients infected with HCV genotype 2 can be treated for a shorter period of time. In a similar study, involving patients infected with HCV genotype 1, good results were demonstrated using a 24-week course of treatment. Patients achieving an RVR and a low viral load $(<600,000 \mathrm{IU} / \mathrm{mL})$, treated for this short period of time, presented a high cure rate (89\%), confirming the hypothesis that, even in patients infected with the more difficult to treat genotypes, it is possible that treatment can be shortened if patients are adequately selected regarding short-term regimens.

Although further studies are necessary to confirm the efficacy of less prolonged therapies for the treatment of chronic hepatitis $\mathrm{C}$, these current studies have already indicated that it is possible to use this strategy for patients achieving an RVR, significantly improving treatment compliance and quality of life of the patients, as well as reducing the frequency of side effects and increasing the cost-effectiveness ratio. Table 2 summarizes the current recommendations for the treatment of chronic hepatitis $\mathrm{C}$, showing the recommended medications and doses, together with treatment durations and responses. 


\section{Contraindications to and Side Effects of Treatment}

Absolute contraindications to the PEG-IFN- $\alpha$ and ribavirin treatment include pregnancy, breastfeeding and hypersensitivity to either drug. Relative contraindications, due to potential side effects are as follows: decompensated liver disease (jaundice, ascites, hepatic encephalopathy, severe coagulopathy, etc.); neuropsychiatric, pulmonary, cerebrovascular or coronary diseases; severe autoimmune diseases; malignant neoplasms; convulsions; and history of solid organ transplants. Patients addicted to injection drugs, as well as chronic alcoholics, should be advised to abandon the habit (for at least six months) prior to the beginning of the antiviral therapy. Patients diagnosed with anemia, leukopenia and platelet reduction should be treated with care, and hematological parameters should be closely monitored throughout the treatment.

Side effects of interferon and ribavirin affect practically all of the patients. Table 3 shows these side effects, which are organized by their frequency.

The most common side effects are fatigue, myalgia, psychological alterations (depression, anxiety, insomnia, and irritability) and worsening of hematological parameters (anemia, platelet reduction, and leukopenia). Ribavirin induces hemolytic anemia, which frequently requires dose reduction. In addition, ribavirin is teratogenic, which requires strict contraceptive control during treatment. Only 1\% to $2 \%$ of patients will develop severe side effects, requiring the interruption of one or both medications. Recombinant erythropoietin (for anemia) and filgrastim (for neutropenia) should be routinely used for the control of treatment-induced cytopenias. Patients suffering from depression or mood disorders can use antidepressants or anxiolytics, with variable success.

\section{References}

1. Hoofnagle J.H., Seef L.B. Peginterferon and Ribavirin for chronic hepatitis C. New England Journal of Medicine 2006;355:244451.

2. Strader D.B., Wright T., Thomas D.L., Seef L.B. Diagnosis, management and treatment of hepatitis C. Hepatology 2004;4:1147-71.

3. Iacobellis A., Siciliano M., Perri F., et al. Peginterferon alfa-2b and ribavirin in patients with hepatitis $C$ virus and decompensated cirrhosis: a controlled study. Journal of Hepatology 2007;46:206-12.

4. Bruno S., Stroffolini T., Colombo M., et al. Sustained virological response to Interferon a is associated with improved outcome in HCV-related cirrhosis: a retrospective study 2007;45:579-87.

5. Schiff E.R. Emerging strategies for pegylated interferon combination therapy. Gastroenterology \& Hepatology 2007;4(1):517-21.

6. Navasa M., Forns X. Antiviral therapy in HCV decompensated cirrhosis: to treat or not to treat? Journal of Hepatology 2007;46:185-8.

7. Everson G.T., Hoefs J.C., Seef L.B., et al. Impact of disease severity on outcome of antiviral therapy for chronic hepatitis C: lessons from the HALT-C trial. Hepatology 2006;44:1675-84.

8. Tan J., Lok A.S.F. Update on viral hepatitis: 2006. Current Opinion in Gastroenterology 2007;263-7.
Table 3. Side effects of interferon and ribavirin

\begin{tabular}{ll}
\hline \multicolumn{1}{c}{ Side effects } & \\
\hline Interferon $\alpha$ (includingPEG-IFN) & Ribavirin \\
\hline Flu symptoms (greater withPEG-IFN- $\alpha$ 2a) & Hemolytic anemia \\
Neutropenia & Fatigue \\
Thrombocytopenia & Pruritus \\
Depression, acute psychosis, & Skin rash \\
suicide attempts & Sinusitis \\
Irritability & Fetalmalformations \\
Visual disorders & Gout \\
Fatigue, myalgia & \\
Hypothyroidism/hyperthyroidism & \\
Headache & \\
Nausea, vomiting & \\
Itching & \\
Fever, weight loss & \\
Hearing loss & \\
Alopecia & \\
Pulmonary interstitial fibrosis & \\
Angina/myocardial infarction & \\
Bacterial infections (in cirrhotic patients) & \\
\hline
\end{tabular}

PEG-IFN=pegylated interferon.

9. Hadzyiannis S.J., Sette H., Morgan T.R., et al. Peginterferon a 2a and Ribavirin combination therapy in chronic hepatitis C. Annals of Internal Medicine 2004;140:346-55.

10. Pawlotsky J.-M. Current and future concepts in hepatitis C therapy. Seminars in Liver Disease 2005;25:72-83.

11. Mangia A., Santoro R., Minerva N., et al. Peginterferon alfa 2b and Ribavirin for 12 vs 24 weeks in HCV genotype 2 or 3. The New England Journal of Medicine 2005;325:2609-17.

12. Zenzem S., Buti M., Ferenci P., et al. Efficacy of 24 weeks treatment with chronic hepatitis $\mathrm{C}$ infected with genotype 1 and low pretreatment viremia. Journal of Hepatology 2006;44:97-103.

13. Delgard O., Bjoro K., Hellum K.B., et al. Treatment with pegylated interferon and ribavirin in $\mathrm{HCV}$ infection with genotype 2 or 3 for 14 weeks: a pilot stydy Hepatology 2004;40:1260-5.

14. Manns M., Waldemeyer H., Cornberg M. Treating viral hepatitis C: efficacy, side effects and complications. Gut 2006;55:1350-9.

15. Davis G.L. Monitoring of viral levels during therapy of hepatitis C. Hepatology 2002;36:5145-51.

16. Manns M.P., McHutchison J.G., Gordon S.C., et al. Peginterferon alfa $2 b$ plus ribavirin for initial treatment of chronic hepatitis C: a randomized trial. Lancet 2001;358:958-65.

17. Hofmann W.P., Zeuzem S., Sarrazin C. Towards individualized antiviral therapy of patients infected with hepatitis $C$ virus genotypes 2 and 3. Hepatology Reviews 2006;3:3-10.

18. Abergel A., Hezode C., Leroy V., et al. Peginterferon alpha-2b plus ribavirin for treatment of chronic hepatitis $\mathrm{C}$ with severe fibrosis: a multicentre randomized controlled trial comparing two doses of peginterferon alpha 2b. Journal of Viral Hepatitis 2006;13:811-20.

19. Hung C.H., Lee C.M., Lu S.N., et al. Long term effect of interferon alpha $2 b$ plus ribavirin therapy on incidence of hepatocellular carcinoma in patients with hepatitis $C$ virus-related cirrhosis. Journal of Viral Hepatitis 2006;13:409-14.

20. EASL International Consensus Conference on hepatitis C. Paris, 26-27 February 1999. Consensus statement. Journal of Hepatology 1999;3151:3-8. 